\title{
Relação entre orientações motivacionais, ansiedade e autoconfiança, e bem-estar subjetivo em atletas brasileiros
}

\author{
Relationship between achievement goals, anxiety and self-confidence, \\ and subjective well-being in Brazilian athletes
}

\author{
M.G. Fernandes, J. Vasconcelos-Raposo, H.M. Fernandes
}

ARTIGO ORIGINAL | ORIGINAL ARTICLE

\begin{abstract}
RESUMO
O presente estudo pretendeu investigar a relação entre orientações motivacionais, ansiedade e autoconfiança e bem-estar subjetivo (afetos positivos e negativos, e satisfação com vida) através da técnica de análise de caminhos. A amostra foi constituída por 169 atletas brasileiros (140 do sexo masculino e 29 do sexo feminino) com idades compreendidas entre os 17 e os 59 anos, de diferentes modalidades desportivas e níveis competitivos. Os questionários TEOSQ, CSAI-2, EBES e SWLS foram aplicados no dia anterior à competição, no local de treino dos atletas. Os principais resultados suportaram parcialmente o papel mediador da ansiedade e autoconfiança na relação entre orientações motivacionais e bem-estar subjetivo. Tendo em consideração índices de modificação do modelo estrutural, foram ainda especificados coeficientes de regressão entre a orientação para o ego e os afetos negativos, e entre a orientação para a tarefa e as dimensões positivas do bem-estar (afetos positivos e satisfação com a vida). Os resultados foram discutidos tendo em contas as implicações teóricas e práticas destas relações.

Palavras-chave: orientações motivacionais, ansiedade competitiva, bem-estar subjetivo, análise de caminhos
\end{abstract}

ABSTRACT

The present study aimed to investigate the relationship between achievement goals, anxiety, selfconfidence and subjective well-being (positive and negative affect, and satisfaction with life) through path analysis. The sample consisted of 169 Brazilian athletes (140 males and 29 females), aged between 17 and 59 years, of different sports and competitive levels. The questionnaires TEOSQ, CSAI-2, EBES and SWLS were applied the day before the competition, on-site training of athletes. Main results partially demonstrated that anxiety and self-confidence mediated the relationship between motivational orientations and subjective well-being. Based on modification indices of the structural model, regression coefficients were specified between ego orientation and negative affect, and between task orientation and the positive dimensions of well-being (positive affect and satisfaction). Results were discussed taking into account the theoretical and practical implications of these relationships.

Keywords: achievement goals, competitive anxiety, subjective well-being, path analysis

Submetido: 18.02.2012 | Aceite: 17.09.2012

Marcos Gimenes Fernandes. Departamento de Ciências da Saúde, Universidade Estadual de Santa Cruz, Ilhéus, Bahia, Brasil.

José Vasconcelos-Raposo. Universidade de Trás-os-Montes e Alto Douro, Vila Real, Portugal.

Helder M. Fernandes. Cidesd - Centro de Investigação em Desporto, Saúde e Desenvolvimento Humano, UTAD, Vila Real, Portugal.

Endereço para correspondência: Marcos Gimenes Fernandes, Departamento de Ciências da Saúde, Universidade Estadual de Santa Cruz, Campus Soane Nazaré de Andrade, Rodovia Jorge Amado, Km 16, Bairro Salobrinho, CEP 45662-900, Ilhéus, Bahia, Brasil.

E-mail: gimenesfmarcos@gmail.com 
Os estudos da ansiedade competitiva (AC) e seus principais fatores de influência têm sido frequentemente examinados na Psicologia do Desporto (Woodman \& Hardy, 2001), usando um modelo multidimensional em que a AC é constituída por uma dimensão cognitiva e uma dimensão somática (Martens, Vealey, \& Burton, 1990). Segundo esses autores, a ansiedade cognitiva engloba as expectativas negativas, por parte do atleta, acerca do seu desempenho, enquanto a ansiedade somática se refere aos aspetos fisiológicos da experiência e manifestada através da função autonômica (aumento da frequência cardíaca, sudorese, dilatação da pupila, etc). Em 1990, Martens, Vealey e Burton desenvolveram o Competitive State Anxiety Inventory (CSAI-2), um teste psicométrico específico para mensurar a AC em competições esportivas. Este se tornou um dos principais instrumentos de avaliação da ansiedade-estado de atletas, proporcionando uma pletora de estudos com objetivo de examinar a ansiedade no contexto competitivo (Jones, 1995).

Posteriormente, Cox, Martens e Russell (2003) aplicaram o CSAI-2 numa amostra de calibração constituída por 503 atletas com o objetivo de refinar o instrumento. A análise inicial indicou índices de ajustamento (validade fatorial) fracos. Na fase seguinte, eliminaram 10 itens, resultando num modelo de 17 itens (CSAI-2R), mantendo a estrutura dos três fatores originais. Esta reespecificação foi aplicada numa amostra de validação com 331 atletas, tendo-se verificado bons índices de ajustamento $(\mathrm{CFI}=.95, \mathrm{NNFI}=.94$ e RMSEA $=$ .054). Perante estes resultados, os autores recomendaram que profissionais e pesquisadores interessados em avaliar a ansiedade competitiva no contexto desportivo, utilizassem o instrumento CSAI-2R em substituição do CSAI-2 original. Recentemente, no contexto desportivo brasileiro, Coelho, Vasconcelos-Raposo e Mahl (2010) validaram a versão CSAI-2R numa amostra de 529 futebolistas e Fernandes, Vasconcelos-Raposo e Fernandes (no prelo) testaram diversos modelos do CSAI-2 sugeridos previamente na literatura, numa amostra de atletas de diferentes modalidades esportivas $(n=375)$. Os resultados das análises fatoriais confirmatórias indicaram melhores índices de ajustamento para o modelo do CSAI-2R, suportando a utilização dessa versão reduzida para avaliar a ansiedade competitiva em atletas brasileiros.

Com o objetivo de aprofundar as questões relacionadas à $\mathrm{AC}$, vários estudos têm investigado a relação entre a AC e outras variáveis cognitivas e motivacionais (Ntoumanis \& Biddle, 1998). De forma geral, o estudo da motivação tem sido objeto de diversas investigações na Psicologia. Especificamente, a psicologia educacional tem-se ocupado da compreensão de processos denominados de objetivos de realização/orientações motivacionais (Ames, 1992; Dweck, 1986). Baseados na teoria social-cognitiva, Dweck e Leggett (1988) e Nicholls (1989), defendem que a perceção de orientações cognitivas para objetivos (orientação motivacional) pode ser subdivida em dois tipos de perspetivas. No caso da orientação para o ego, os indivíduos avaliam o seu desempenho através da comparação com outros indivíduos (referencial normativo). Por outro lado, o indivíduo pode avaliar o seu desempenho voltando-se para a aprendizagem e o seu aperfeiçoamento (autorreferência), o qual é denominado como orientação para a tarefa. Uma ampla evidência empírica suporta a validade da utilização destes objetivos de realização no contexto desportivo (e.g., Duda, Chi, Newton, Walling, \& Catley, 1995; Roberts, 2001), verificando-se primordialmente a utilização do instrumento Task and Ego Orientation in Sport Questionnaire (TEOSQ; Duda \& Nicholls, 1992).

Ntoumanis e Biddle (1998) sugeriram a hipótese que o indivíduo com dominância de orientação para o ego tende a ser mais suscetível a elevados níveis de ansiedade competitiva. Esta afirmação foi baseada na explicação de Roberts (1992), que entende que ganhar e 
perder no desporto são situações instáveis e incontroláveis e, portanto podem criar estados afetivos aversivos ao rendimento. Por outro lado, atletas com orientação para a tarefa não estariam tão suscetíveis a estados adversos como, por exemplo, a ansiedade competitiva, dado possuírem padrões internos de desempenho (independente do resultado) e o objetivo pretendido por estes ser relativamente controlável. Contudo, existem outros estudos que não confirmaram estas relações teoricamente previstas. No estudo de Ntoumanis e Biddle (1998) a orientação para a tarefa não se relacionou significativamente com as dimensões do CSAI-2, enquanto a orientação para o ego associou-se negativamente com a ansiedade cognitiva $(r=-.19)$ e positivamente com a autoconfiança $(r=.25)$. Por sua vez, Rodrigues, Lázaro, Fernandes e Vasconcelos-Raposo (2009) verificaram, num estudo com alpinistas, uma relação positiva e significativa entre a orientação para o ego e a ansiedade cognitiva $(r$ $=.33$ ), enquanto a orientação para a tarefa não se relacionou significativamente com as dimensões do CSAI-2.

Uma possível explicação para esta inconsistência de resultados é a de que os atletas podem diferir na interpretação cognitiva dos seus sintomas de ansiedade, percecionando-os como facilitadores ou dificultadores do seu desempenho em função das suas características individuais (Jones, Hanton, \& Swain, 1994; Jones \& Swain, 1995). Segundo estes últimos autores as diferenças individuais na interpretação dos sintomas de ansiedade podem estar associadas a variáveis denominadas de afetos positivos e afetos negativos (Watson \& Clarke, 1984; Watson, 1988). Para estes autores, afetos positivos e negativos são estruturas afetivas que refletem uma tendência individual para exibir estados de humor adaptativo ou aversivo. Afetos positivos associam-se a estados de entusiasmo e motivação caracterizados por completa concentração e aceitação de desafios, enquanto afetos negativos dizem respeito a uma visão geral para estados relacionados a situações de estresse caracterizadas por medos, preocupações e nervosismo. Jones, Swain e Harwood (1996) reportaram correlações positivas significativas entre a ansiedade cognitiva e somática e os afetos negativos. Estes autores ainda relataram que indivíduos com altos níveis de afetos negativos tendem a experimentar altos níveis de ansiedade cognitiva e somática. Recentemente, Mellalieu e Hanton (2008), reportaram correlações positivas significativas entre os afetos negativos e a ansiedade cognitiva e somática, bem como entre os afetos positivos e a autoconfiança. Por outro lado, os mesmos autores relataram correlações negativas significativas entre a autoconfiança e os afetos negativos. No entanto, a evidência científica ainda não esclareceu a relação causal entre estas dimensões. Robazza, Bortoli, Nocini, Moser e Arslan (2000) demonstraram que a ansiedade-traço atua como preditora do afeto negativo quer numa situação de avaliação normativa, quer ideográfica. Um estudo realizado por Cerin (2004) sugeriu que os afetos positivos podem atuar como preditores da direção da ansiedade cognitiva e somática.

De acordo com Diener, Emmons, Larsen e Griffin (1985), os afetos positivos e negativos podem ser integrados num modelo multidimensional de bem-estar subjetivo, no qual estes fatores são integrados numa dimensão afetiva a par de uma dimensão cognitiva (satisfação com a vida). Gouveia, Milfont, Fonseca e Coelho (2009) demonstraram numa grande amostra brasileira que a satisfação com a vida se correlaciona positivamente com os afetos positivos e negativamente com os afetos negativos. Contudo, uma análise extensiva da literatura especializada da Psicologia do Desporto revela que a dimensão cognitiva do modelo de bem-estar subjetivo tem sido negligenciada. Embora alguns estudos indiquem possíveis associações entre a ansiedade competitiva e os afetos (positivos e negativos), o mesmo não se verifica para a satisfação com a vida. 
Assumindo que a dimensão da participação e competição desportiva constitui uma importante e valorizada dimensão da vida dos atletas, torna-se fulcral compreender quais as possíveis influências que as orientações motivacionais e as emoções pré-competitivas exercem no seu bem-estar e qualidade de vida.

Para os propósitos do presente estudo tomamos como referência o estudo de Robazza et al. (2000), uma vez que este se apresenta como aquele que provavelmente reflete melhor o tipo de compreensão que atletas, treinadores e alguns psicólogos que intervêm na preparação de atletas têm sobre a relação entre ansiedade e rendimento desportivo. Com base nas propostas existentes na literatura foi concebido um modelo teórico que estabelece o tipo de relação entre orientações motivacionais, ansiedade, bem-estar subjetivo e satisfação com a vida. Assim, a teoria preconiza a existência de correlações negativas entre orientação para a tarefa e ansiedade cognitiva, somática e afetos negativos e uma relação positiva com a autoconfiança, afetos positivos e satisfação com a vida. De acordo com a teoria proposta, estas relações desenvolvem-se através da acumulação de vivências emocionais em situações competitivas tipificadas por estados de ansiedade (cognitiva e somática) e de autoconfiança, que com o passar do tempo se repercutem no bem-estar subjetivo dos sujeitos enquanto atletas. Assim, quanto mais intensa for a orientação para a tarefa, menores serão os níveis de ansiedade cognitiva e somática e maiores os de autoconfiança. Relativamente à orientação para o ego, deverão surgir correlações positivas com a ansiedade cognitiva e somática e negativa com a autoconfiança. Por sua vez, quanto maiores forem os níveis de ansiedade cognitiva menores serão os indicadores de afetos positivos e maiores os de afetos negativos. Já no que refere à autoconfiança esta deverá estar positivamente associada a elevados níveis de afetos positivos e com baixos níveis de afetos negativos. Como consequência destas relações será de prever que a ansiedade cognitiva se relacione negativamente com a satisfação com a vida assim como positivamente com a autoconfiança.

Diante das evidências e limitações empíricas apresentadas, o objetivo principal do presente estudo foi investigar a relação entre orientações motivacionais, ansiedade e autoconfiança competitiva, e bem-estar subjetivo (afetos positivos e negativos, e satisfação com a vida) em atletas brasileiros, através da análise de caminhos (path-analysis). Deste modo, a definição das relações causais dos modelos estruturais baseou-se em pressupostos teóricos e empíricos (e.g., Ames, 1992; Duda, 1989; Duda \& Nicholls, 1992; Ntoumanis \& Biddle, 1998; Robazza et al., 2000). De um modo geral, testou-se o papel central e fulcral das dimensões do CSAI-2R (ansiedade cognitiva, ansiedade somática e autoconfiança) na mediação entre as orientações motivacionais e o bem-estar subjetivo dos atletas. Um primeiro modelo (Modelo 1, ver Figura 1) assumiu que as três dimensões do CSAI-2R atuam a um mesmo nível intermédio entre as orientações motivacionais e as dimensões afetiva (afetos positivos e negativos) e cognitiva (satisfação com a vida) do bem-estar. Por sua vez, o Modelo 2 (ver Figura 2) baseia-se no estudo de Ntoumanis e Biddle (1998), o qual sugere que o efeito das orientações motivacionais na ansiedade cognitiva e somática é mediado pela dimensão autoconfiança.

\section{MÉTODO}

\section{Amostra}

A amostra, do tipo não probabilística e intencional, foi composta por 169 atletas (140 do sexo masculino e 29 do sexo feminino), de diferentes modalidades desportivas, com idades compreendidas entre os 17 e os 59 anos $(M=$ 31.70, $D P=10.32$ ). Os atletas tinham entre 1 e 42 anos de prática esportiva $(M=9.14, D P=$ 9.33) e entre 1 e 42 anos de experiência competitiva $(M=7.25, D P=8.67)$. Não se verificaram diferenças significativas entre sexos para as variáveis idade, tempo de prática despor- 


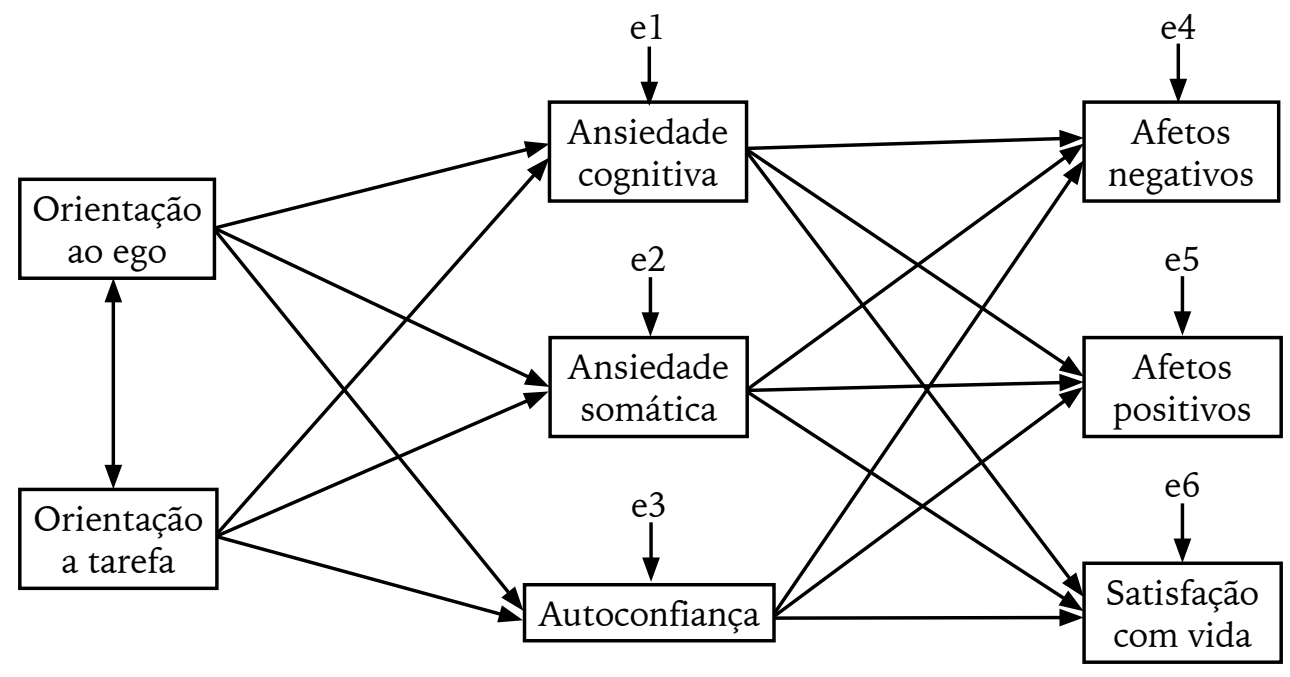

Figura 1. Modelo hipotético 1

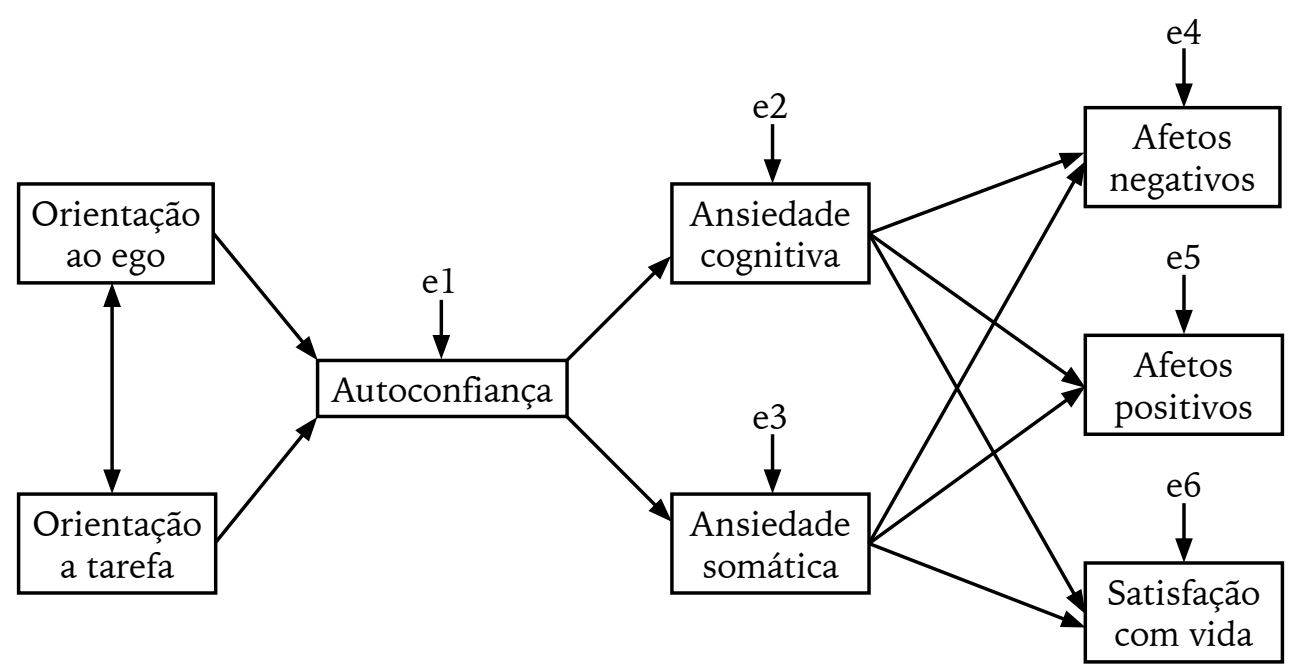

Figura 2. Modelo hipotético 2 (Ntoumanis \& Biddle, 1998)

tiva e anos de competição $(p>$.05). Quando analisada a modalidade desportiva praticada obteve-se a seguinte distribuição: futebol (4\%), basquetebol (1\%), judo (1\%), jiu-jitsu (40\%), karaté $(2 \%)$, corrida (17\%), surfe (3\%), natação (2\%), artes-marciais mistas (10\%), remo $(38 \%)$ e taekwondo (4\%). De um modo geral, $73 \%$ dos atletas praticavam modalidades esportivas individuais, enquanto $27 \%$ dos atletas praticavam modalidades esportivas coletivas. Relativamente ao nível competitivo, 58.5\% dos atletas afirmaram participar em competições de nível regional, enquanto os restantes $41.5 \%$ dos atletas afirmaram participar em competições nacionais.

\section{Instrumentos}

Os atletas responderam a uma versão brasileira (Fernandes, Vasconcelos-Raposo, \& Fernandes, no prelo) do CSAI-2R (Cox, Martens, \& Russell 2003). Este é um instrumento constituído por 17 itens, agrupados em três fatores, da seguinte forma: os itens $2,5,8$, 11 e 14 pertencem ao fator ansiedade cognitiva; $1,4,6,9,12$, 15 e 17 à ansiedade somática; e, $3,7,10,13$ e 16 à autoconfiança. As afirmações foram respondidas de acordo com uma escala do tipo Likert de quatro pontos ( 1 = nada a 4 = muito). É possível calcular um escore para cada dimensão, através da soma das respostas dos itens de cada fator dividida pelo respectivo 
número de itens, podendo os valores variar entre 1 e 4.

Os atletas também responderam a uma versão traduzida e adaptada para a língua portuguesa (Fernandes \& Vasconcelos-Raposo, 2010) do Task and Ego Orientation in Sport Questionnaire (TEOSQ; Duda \& Nicholls, 1992). Este instrumento é constituído de 13 itens, agrupados em dois fatores: orientação para tarefa - itens 2, 5, 7, 8, 10, 12 e 13; e orientação para o ego - itens 1, 3, 4, 6, 9 e 11. As afirmações foram respondidas de acordo com uma escala do tipo Likert de cinco pontos $(1=$ discordo completamente a 5 = concordo completamente). O escore para cada dimensão foi calculado através da soma das respostas dos itens de cada fator divididos pelo respetivo número de itens.

Para a mensuração do bem-estar subjetivo, os atletas responderam a dois questionários. Para a mensuração dos afetos positivos e afetos negativo, foram utilizadas as respetivas dimensões (47 itens) da Escala de Bem-Estar Subjetivo (Albuquerque \& Tróccoli, 2004). Os itens são agrupados da seguinte forma: os itens 3,4 , $6,7,10,11,14,15,16,18,21,22,24,25,26,29$, $37,39,41,42$ e 43 pertencem ao fator afetos positivos; $1,2,5,8,9,12,13,15,17,20,23$, $27,28,30,31,32,33,34,35,36,38,40,44$, 45,46 e 47 ao fator afetos negativos. As afirmações foram respondidas de acordo com uma escala do tipo Likert de cinco pontos $(1=$ nem um pouco a $5=$ extremamente). O escore para cada dimensão foi calculado através da soma das respostas dos itens daquele fator divididos pelos números de itens do fator, podendo os valores variar entre 1 e 5 . Para a mensuração da satisfação com a vida, os atletas responderam à versão brasileira (Gouveia, Milfont, Fonseca, \& Coelho, 2009) da Satisfaction With Life Scale (Diener et al.,1985). Este instrumento é constituído por uma única dimensão com cinco itens. As afirmações foram respondidas de acordo com uma escala do tipo Likert de sete pontos $(1=$ discordo totalmente a $7=$ concordo totalmente). Os escores são calculados somando os números correspondentes às respostas escolhidas pelo sujeito, podendo variar de 5 a 35 .

\section{Procedimentos}

Após a devida autorização dos organizadores das competições e dos técnicos para a coleta de dados, os atletas foram informados dos objetivos da investigação e assinaram um termo de consentimento livre e esclarecido (TCLE), sendo garantidos o anonimato e confidencialidade de todos os dados recolhidos. Aos atletas menores de 18 anos foi solicitado que um responsável maior de idade assinasse o TCLE.

Tendo em consideração os objetivos do estudo, alguns cuidados ao nível da recolha de dados foram acautelados, uma vez que as previsões teóricas requerem que sejam avaliados traços de ansiedade cognitiva e somática assim como de autoconfiança. Para este efeito foi solicitado aos atletas que constituíram a amostra que ao responderem aos questionários tivessem em consideração o seu histórico de respostas afetivas nos momentos que antecedem a competição. Esta preocupação foi reforçada pelo fato de que os dados serem recolhidos em contexto de treino e não pré-competitivo.

O presente estudo foi aprovado pelo Comitê de Ética em Pesquisa (Protocolo 425/2010) da Universidade Estadual de Santa Cruz (UESC), de acordo com a Resolução CNS/MS n. 196/1996.

\section{Análise Estatística}

Inicialmente procedeu-se a uma estatística descritiva (média e DP) das dimensões analisadas. Coeficientes de assimetria e curtose foram computados para efeitos de análise de normalidade univariada. A confiabilidade das escalas foi avaliada através da computação de alphas de Cronbach. A associação entre variáveis foi determinada através de coeficientes de correlação de Pearson. Essas análises estatísticas foram realizadas usando o SPSS v16.

A técnica de análise de caminhos foi realizada usando o programa AMOS v16.0. Este 
método de modelação de equações estruturais tem sido recentemente usado em estudos que visam testar a relação entre variáveis psicológicas no contexto desportivo (ex., Heazlewood \& Burke, 2011; Tello, Martínez, Núñez, \& Calvo, 2010), permitindo o desenvolvimento e o teste de complexas teorias sociais (Duncan \& Stoolmiller, 1993). A modelação de equações estruturais tem a vantagem sobre a técnica de análise de regressão de possibilitar o teste simultâneo da relação entre duas ou mais variáveis na presença e potencial influência de outras variáveis (Ntoumanis \& Biddle, 1998).

Dessa forma, após a especificação e estimação dos modelos, a sua adequação foi avaliada por um conjunto de índices de ajustamento/adequação. O valor de $\chi^{2}$ (Qui-quadrado) indica ajustamento quando o valor não é significativo $(p>.05)$. No entanto, esse teste é sensível ao tamanho da amostra, ou seja, em amostras numerosas o valor tende a ser significativo, embora o modelo possa estar ajustado aos dados. Jöreskog e Sörbom (1989) sugeriram uma razão do Qui-quadrado pelos graus de liberdade ( $d f$ ), representado por $\chi^{2} / d f$, pelo que Ullman (2001) sugere valores abaixo de 2.0 como aceitáveis. Adicionalmente, foram utilizados os seguintes índices de ajustamento: a) CFI (Comparative Fit Index) e GFI (Goodness Fit Index) podendo os seus valores variar de 0 a 1. Segundo Bentler e Bonnet (1980), valores acima de .90 representam um ajuste adequado para o modelo. Mais recentemente, $\mathrm{Hu}$ e Bentler (1999) sugeriram um ponto de corte de .95 como indicativo de um bom ajustamento do modelo; b) RMSEA (Root Mean Square Error of Approximation), em que valores menores que .08 indicam uma adequação aceitável (Browne \& Cudeck,1993), embora Hu e Bentler (1999) tenham sugerido um ponto de corte de .06; e, c) AIC (Akaike Information Criterion) que indica a parcimônia e simplicidade do modelo através do menor valor entre os modelos testados.

\section{RESULTADOS}

Análise descritiva e correlacional

A análise descritiva preliminar dos dados indicou que os coeficientes de normalidade das variáveis variaram entre -.79 e 1.20, indicando valores associados a uma distribuição aproximadamente normal (Kline, 2010). A Tabela 1 apresenta as médias, desvios padrões, índices de consistência interna (alpha de Cronbach) e correlações de todas as variáveis mensuradas.

A análise descritiva das dimensões indica que os atletas revelaram níveis superiores de orientação para a tarefa (em comparação à orientação para o ego), autoconfiança (em comparação à ansiedade cognitiva e somática) e afetos positivos (em comparação aos afetos

Tabela 1.

Médias, desvio-padrão, confiabilidade e correlações de Pearson

\begin{tabular}{lcccccccc}
\hline Variáveis & 1 & 2 & 3 & 4 & 5 & 6 & 7 & 8 \\
\hline 1. Orientação ao ego & $(.79)$ & .02 & .25 & .23 & -.05 & -.16 & .33 & .01 \\
2. Orientação à tarefa & & $(.77)$ & -.07 & -.01 & .32 & .38 & .02 & .21 \\
3. Ansiedade cognitiva & &. & $(.82)$ & .50 & -.39 & -.28 & .50 & -.21 \\
4. Ansiedade somática & & & & $(.77)$ & -.14 & -.21 & .48 & -.23 \\
5. Autoconfiança & & & & & $(.85)$ & .46 & -.18 & .18 \\
6. Afeto positivo & & & & & & $(.92)$ & -.35 & .29 \\
7. Afeto negativo & & & & & & & $(.93)$ & -.28 \\
8. Satisfação com a vida & & & & & & & & $(.75)$ \\
\multicolumn{1}{c}{ Média } & 2.33 & 4.31 & 2.10 & 2.00 & 2.94 & 3.71 & 1.80 & 26.89 \\
\multicolumn{1}{c}{ Desvio padrão } & 0.80 & 0.51 & 0.69 & 0.55 & 0.60 & 0.59 & 0.59 & 4.70 \\
\hline
\end{tabular}

Nota: Correlações acima de $|.15|$ são $\mathrm{p}<.05$; Correlações acima $|.20|$ são $\mathrm{p}<.01$. Os coeficientes de confiabilidade (alpha de Cronbach) são apresentados entre parênteses na diagonal. 
negativos). Os valores de satisfação com a vida podem ser considerados moderados a elevados (variação possível entre 5 e 35). Todas as escalas revelaram bons índices de fiabilidade $(\alpha>.70)$.

De um modo geral, as correlações obtidas estão de acordo com as hipóteses teóricas. A correlação não significativa entre a orientação para o ego e para a tarefa suporta a ortogonalidade conforme o argumento de Nicholls (1989). A orientação para o ego associou-se positivamente com a ansiedade cognitiva, a ansiedade somática e os afetos negativos, e negativamente com os afetos positivos. A orientação para a tarefa correlacionou-se positivamente com a autoconfiança e as dimensões positivas do bem-estar. A ansiedade cognitiva correlacionou-se positivamente com a ansiedade somática e afetos negativos, verificando-se correlações negativas com a autoconfiança, afetos positivos e satisfação com a vida. A ansiedade somática relacionou-se positivamente com os afetos negativos e negativamente com as dimensões positivas do bem-estar. A autoconfiança associou-se positivamente com os afetos positivos e satisfação com a vida e negativamente com afetos negativos. As relações entre as dimensões do bem-estar subjetivo confirmaram os pressupostos teóricos.

\section{Análise de caminhos}

$\mathrm{Na}$ análise de caminhos adotámos a estratégia de testar simultaneamente os dois modelos previamente definidos (ver Figuras 1 e 2). Tendo em consideração as correlações significativas verificadas entre dimensões do mesmo nível (variáveis do CSAI-2 e dimensões do bem-estar subjetivo) foi permitido que os erros de medição dessas variáveis se correlacionassem no mesmo nível dos modelos.

Na Tabela 2 são apresentados os índices de ajustamento obtidos.

A análise dos modelos testados indica que o modelo 1 obteve melhores índices de ajustamento em todos parâmetros em comparação ao modelo 2 proposto por Ntoumanis e Biddle
Tabela 2.

Indices de ajustamento das AFC aos diferentes modelos

\begin{tabular}{lcccccc}
\hline & $\chi^{2}$ & $\chi^{2} / d f$ & CFI & GFI & RMSEA & AIC \\
\hline Modelo 1 & 33.53 & 5.59 & .891 & .955 & .165 & 93.53 \\
Modelo 2 & 79.91 & 6.15 & .734 & .904 & .175 & 125.91 \\
\hline Nota: Todos os valores de $\chi^{2}$ são significativos $(p<.01)$
\end{tabular}

(1998). Como tal, procedeu-se à análise mais detalhada dos parâmetros especificados para o modelo 2 e respectivos índices de modificação. Os resultados indicaram os seguintes pares de relações/regressões não significativas $(p>$.05): orientação para o ego e orientação para a tarefa; orientação para o ego e autoconfiança; orientação para a tarefa e ansiedade cognitiva; orientação para a tarefa e ansiedade somática; ansiedade cognitiva e afetos positivos; ansiedade cognitiva e satisfação com a vida; autoconfiança e afetos negativos; e autoconfiança e satisfação com a vida. A relação entre os erros de medição das variáveis ansiedade somática e autoconfiança também não se verificou significativa. Estes parâmetros foram eliminados da seguinte revisão do modelo. Adicionalmente, os índices de modificação suportaram os coeficientes de correlação obtidos entre as orientações motivacionais e as dimensões do bem-estar, sendo adicionados as seguintes regressões diretas: orientação para o ego como preditora dos afetos negativos e orientação para a tarefa como preditora dos afetos positivos e da satisfação com a vida.

Este conjunto de re-especificações resultou num modelo com um ótimo ajuste: $\chi^{2}=12.68$, $p=.393, \chi^{2} / d f=1.06, \mathrm{CFI}=.997, \mathrm{GFI}=.982$, RMSEA $=.018$ e AIC $=60.68$. As estimativas dos parâmetros testados são apresentados na Figura 3. As correlações entre erros residuais variaram entre -.34 e $.40(p<.05)$. Adicionalmente testou-se o efeito direto das orientações motivacionais do bem-estar, através da eliminação das regressões entre orientações e dimensões do CSAI-2. Contudo, os resultados obtidos indicam que estas alterações traduziram-se num pior ajuste aos dados: $\chi^{2}=44.50$, $p=.000, \chi^{2} / d f=2.97, \mathrm{CFI}=.883, \mathrm{GFI}=.940$, $\mathrm{RMSEA}=.108$ e $\mathrm{AIC}=86.50$. 


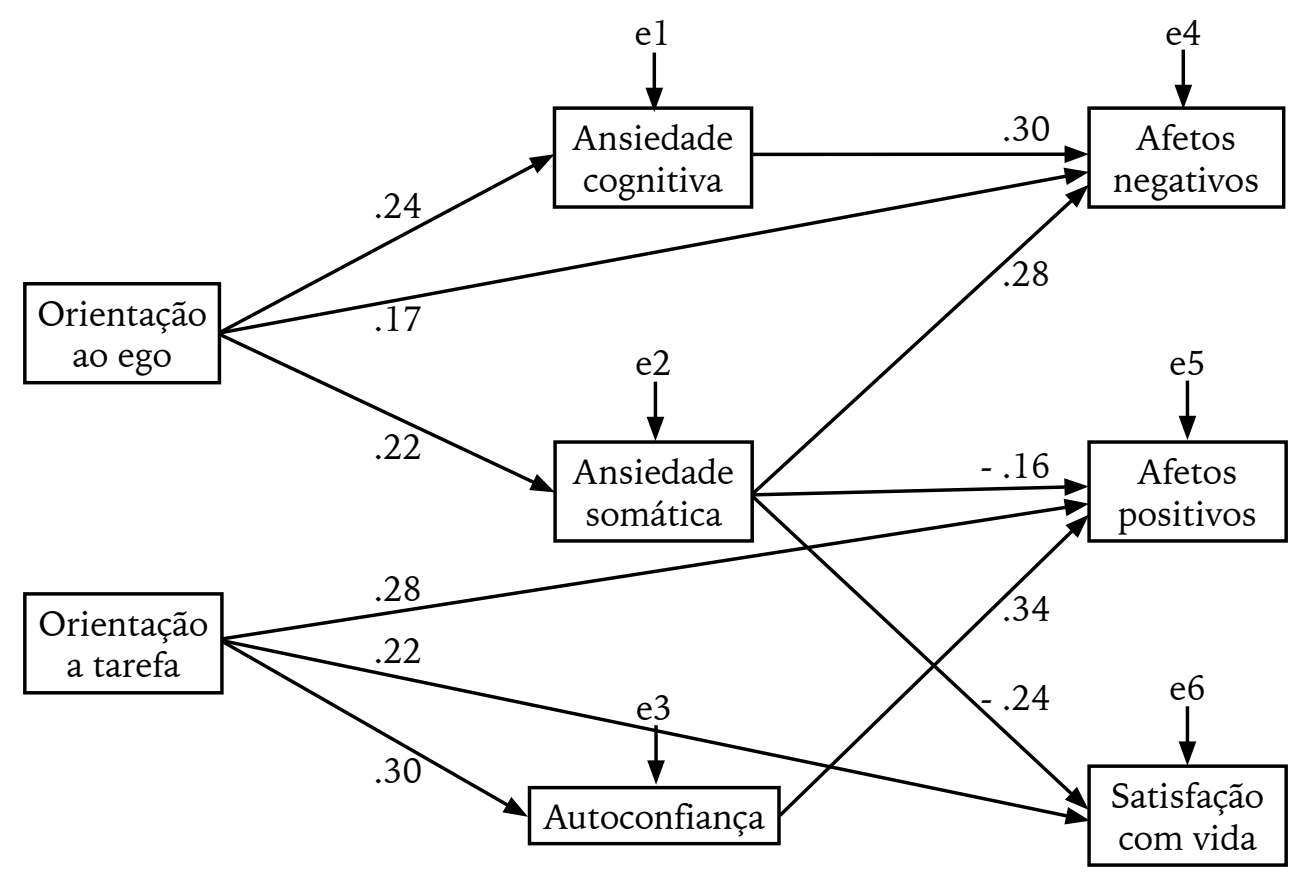

Figura 3. Modelo 1 (revisão final)

Nota: Todos os parâmetros da análise de caminhos são significativos a $p<.05$. Não foram apresentados as correlações entre erros de medição

\section{DISCUSSÃO}

O presente estudo pretendeu investigar a relação entre orientações motivacionais (orientação para a tarefa e orientação para o ego), ansiedade competitiva (ansiedade cognitiva e ansiedade somática), autoconfiança e bem-estar subjetivo (afetos positivos e negativos, e satisfação com vida) no contexto competitivo brasileiro, através da análise de caminhos. Tanto quanto a literatura nos permite constatar este estudo é pioneiro a analisar as relações entre as orientações motivacionais, ansiedade, autoconfiança bem-estar subjetivo e é provavelmente o único que recorre à técnica de análise de caminhos por modelação de equação estrutural neste contexto. Em concordância com estudos que investigaram o impacto da orientação para a tarefa e para o ego sobre a ansiedade competitiva, os sintomas da ansiedade cognitiva e somática e a autoconfiança foram avaliados como mediadores entre orientações motivacionais e dimensões do bem-estar subjetivo (afetos positivos e negativos, e satisfação com a vida), sendo apresentada uma proposta teórica a ser sujeita a comprovação empírica.

\section{Orientações cognitivas e ansiedade competitiva}

O modelo de equação estrutural testou a hipótese de predição da orientação para a tarefa e para o ego sobre a ansiedade cognitiva, ansiedade somática e autoconfiança. Neste primeiro nível do modelo, a orientação para o ego evidenciou regressões significativas sobre a ansiedade cognitiva e somática. De certa forma, estes resultados estão em concordância com a afirmação de Roberts (1992), que relata que os atletas com sua orientação motivacional voltada para o ego estão mais suscetíveis a experimentarem ansiedade cognitiva e somática, assim como com os resultados de Rodrigues et al. (2009), que reportaram correlações positivas significativas entre ansiedade cognitiva e orientação para o ego. Por outro lado, a orientação para a tarefa teve um impacto significativo sobre a autoconfiança, o que está de acordo 
com evidências empíricas anteriores (ex., Hall, Kerr, \& Matthews, 1998). De um modo geral, atletas orientados para a tarefa tendem a centrar-se na aprendizagem e melhoria autorreferenciada, atribuindo o seu sucesso primeiramente ao seu esforço (Fernandes \& Vasconcelos-Raposo, 2010; Vasconcelos-Raposo \& Mahl, 2005), revelando um otimismo acerca das suas reais capacidades e, assim, mostrando-se mais autoconfiantes em situação competitiva. Estes resultados corroboram os princípios teóricos que orientam a prática dos psicólogos do desporto que há muito sabem que uma forma de combater os níveis de ansiedade é promovendo uma orientação cognitiva para a tarefa. Está amplamente em múltiplas áreas da psicologia que a promoção de uma orientação motivacional para a tarefa promove padrões internos de desempenho algo independentes do resultado, controláveis pelos agentes performativos (Roberts, 1992), o que por sua vez promove o desenvolvimento da autoconfiança através de melhorias na perceção de competência e controle efetivo dos níveis indesejados de ansiedade (Martens et al., 1990).

O modelo teórico proposto preconizava regressões que não se verificaram aquando da sua testagem, nomeadamente as relações negativas entre a orientação para o ego e a autoconfiança, e a relação entre orientação para a tarefa e a ansiedade cognitiva e somática.

\section{Ansiedade competitiva e bem-estar subjetivo}

No segundo nível do modelo de equação estrutural verificou-se a hipótese de predição da ansiedade competitiva sobre as dimensões do bem-estar subjetivo. A ansiedade cognitiva e a somática predisseram, positivamente, os afetos negativos, o que está em conformidade com os resultados reportados por Jones et al. (1996). Por outro lado, a autoconfiança exerceu um impacto positivo sobre os afetos positivos, tal como preconizado pela teoria. Este resultado está em concordância com os achados de Mellalieu e Hanton (2008) que sugeriram que os atletas com elevados índices de autoconfiança tendem ter mais afetos positivos. Finalmente, a ansiedade somática predisse negativamente, como esperado, as dimensões positivas do bem-estar (afetos positivos e a satisfação com a vida). De um modo geral, estas relações estão de acordo com estudos anteriores que indicaram que as dimensões da ansiedade competitiva influenciam negativamente o bem-estar de um atleta, podendo esta evidência ser explicada por vários pressupostos. A similaridade da experiência emocional dos sintomas de ansiedade e mal-estar (medos, preocupações e nervosismo) poderá ser percebida pelo atleta de um modo cumulativo, em que as experiências do treino e competição poderão ser transferidas para a forma quotidiana de estar na vida. Em segundo lugar, poderemos também assumir a existência de traços culturais que levam o individuo a exibir estados de humor negativos (Watson \& Clarke, 1984; Watson, 1988), podendo ocorrer nos múltiplos domínios de vida do atleta.

Importa destacar que a ansiedade somática foi a única dimensão do CSAI-2 que foi capaz de predizer significativamente todas as três dimensões do bem-estar subjetivo (afeto positivo, afeto negativo e satisfação com a vida): Desta forma, a importância desta variável no modelo do CSAI-2 (Martens et al., 1990), neste estudo, acaba por ser reforçada, na medida em que constitui uma importante variável preditiva das dimensões do bem-estar subjetivo de atletas brasileiros. Todavia, este é um facto que se apresenta inconsistente com a literatura onde se destaca de forma inequívoca o caráter transitório dos sintomas identificados e que são de todo semelhantes aos espectáveis nas respostas adaptativas do organismo à competição, daí que logo que logo que se dá início ao processo de utilização de recursos energéticos na competição estes sintomas desvaneçam quase de imediato. De acordo com a proposta teórica estas regressões apresentam-se particularmente difíceis de serem interpretadas. Assim 
sendo as regressões relativas à variável ansiedade somática sugerem a necessidade de procurarmos modelos alternativos (Hair, Anderson, Tatham, \& Black, 2005), mas optamos por não testar os modelos alternativos na medida em que o seu número, por si só, requer a realização de um estudo autónomo.

\section{Efeito mediador da ansiedade e da autoconfiança}

O modelo do CSAI-2 (Martens et al., 1990) foi principalmente desenvolvido com base numa orientação para a performance e o resultado competitivo, procurando identificar a relevância do estudo da ansiedade nesse contexto. Estudos prévios têm investigado a influência das orientações motivacionais nas dimensões do CSAI-2 (Ntoumanis \& Biddle, 1999), ou mais recentemente em dimensões do bem-estar subjetivo (Galand, Boudrenghien, \& Rose, in press; Núñez, León, González, \& Martín-Albo, 2011). Contudo, tanto quanto a literatura nos permite conhecer, não existem estudos que tenham analisado a influência mediadora das dimensões do CSAI-2 entre orientações motivacionais e bem-estar subjetivo.

A partir dos dois modelos inicialmente testados, os resultados das análises suportaram parcialmente a hipótese inicial que a ansiedade cognitiva e somática, e a autoconfiança constituem variáveis mediadoras entre as orientações motivacionais e as dimensões do bem-estar subjetivo, a um mesmo nível de ação/ influência. Especificamente, a orientação ao ego através da ansiedade cognitiva impactou nos afetos negativos, assim como impactou sobre os afetos positivos e negativos, e na satisfação com a vida através da ansiedade somática. Por outro lado, a orientação para tarefa exerceu impacto sobre os afetos positivos através da autoconfiança. À luz da teoria estas relações não são explicáveis uma vez que faltam os argumentos e os mecanismos explicativos e necessários para sustentar a validade dos processos que levam à manifestação dos comportamentos aqui em causa. Tal constatação levanta reservas quanto è efectiva relevância da ansiedade somática sugerindo que o que está a ser medido como ansiedade somática seja considerado apenas como uma manifestação dos mecanismos de resposta adaptativa à competição. Este argumento é sustentável nas teorias clássicas da aprendizagem, de que é exemplo a experimentação com cães que Pavlov realizou.

A presente investigação testou ainda um modelo de equação estrutural, proposto por Ntoumanis e Biddle (1998), no qual a orientação para o ego influenciava a ansiedade (cognitiva e somática) através da autoconfiança. As análises do caminho realizadas revelaram índices de ajustamento inaceitáveis para este modelo. Uma explicação plausível para este resultado poderá estar na característica da amostra do presente estudo, que teve uma predominância de atletas de desportos individuais (73\%), diferente da amostra do estudo destes autores onde predominou atletas de desportos coletivos. Estudos anteriores demonstraram que a habilidade preditiva da ansiedade competitiva e da autoconfiança difere entre atletas de desportos individuais e coletivos (Craft, Magyar, Becker, \& Feltz, 2003; Martens et al.,1990;).

\section{Orientações motivacionais e bem-estar subjetivo}

Para além de analisar o efeito mediator das variáveis do CSAI-2, foi também possível verificar que a melhoria do ajustamento do modelo testado contemplava a adição de relações entre as orientações motivacionais e os domínios do bem-estar. O tipo de relações estabelecidas vai de acordo ao teoricamente previsto e que corroboram evidências empíricas anteriores (Galand et al, in press; Núñez et al., 2011). À semelhança do presente estudo, Ntoumanis e Biddle (1999) verificaram existir uma relação direta (predição positiva) e significativa entre orientação para a tarefa e afetos positivos. Estes autores argumentaram que esta relação poderia ter por base o fato de que indi- 
víduos com predominância de orientação para a tarefa se concentram no seu próprio esforço e possuirem padrões de comparação autorreferenciados, o que de acordo com Eliot e Dweck (1988) tende a estar associado a perceções de sucesso e melhora no seu desempenho. Poderá ainda ser argumentado que estas perceções podem estar na base do processo que dê origem de níveis elevados de afetos positivos. Por sua vez, no modelo da presente investigação também se confirmou uma predição positiva significativa entre orientação para a tarefa e satisfação com a vida. Esta relação encontra eco nos argumentos dos teóricos que sugerem que os indivíduos quando intrisicamente motivados (inerente à orientação para a tarefa) quando desejam envolver-se numa determinada atividade o fazem independentemente de qualquer tipo de recompensa, promessas ou até ameaças. Os resultados do nosso estudo vão ao encontro dos que sugerem que estar intrinsecamente motivado contribui positivamente para o bem-estar, assim como para o grau de compromisso necessário para se obter sucesso (Hefferon \& Boniwell, 2011).

A relação positiva entre a orientação para o ego prevista no modelo proposto foi confirmada. Estes resultados, de certa forma, vão de encontro aos achados de Ntoumanis e Biddle (1999). De acordo com o articulado teórico indivíduos com uma orientação para o ego tendem a priorizar as comparações que fazem entre si e os outros. Salmela-Aro et al. (2001) apresentam este tipo de orientação como sendo objectivos focados nos outros e estes tendem a estar pobremente relacionados com o bem-estar e até como o sucesso. No contexto do rendimento desportivo é aceite que atletas com orientação para o ego definem o seu sucesso ou fracasso na comparação com outros indivíduos, estando, assim, mais suscetíveis a sentimentos e comportamentos adversos ao rendimento de alto nível e associados a afetos negativos.

O presente estudo, apesar do ser carácter exploratório sugere que a Psicologia do
Desporto ganharia em expandir os seus referenciais teóricos tendo na Psicologia Positiva (ver Seligman \& Csikszentmihalyi, 2000), provavelmente, uma das suas áreas mais férteis para a sua consolidação teórica e capacidade preditiva.

O presente estudo contém algumas limitações que merecem serem enunciadas, a saber: i) o reduzido tamanho da amostra; ii) a heterogeneidade da amostra do estudo ao nível do tipo de modalidades (individuais e coletivas) e níveis competitivos; e iii) o processo de amostragem não probabilística e intencional, o qual requere uma certa precaução na generalização dos resultados obtidos.

\section{CONCLUSÕES}

Em suma, o presente estudo demonstrou, de forma parcial, que a ansiedade cognitiva está associada aos afetos negativos e que a autoconfiança exerceu um papel mediador na relação entre as orientações motivacionais e os afetos positivos. No entanto, estas associações, tal como constatadas, não são suficientes para validar as propostas teóricas avançadas. Não seria aceitável dar por comprovado um modelo teórico que se alicerçasse em estados transitórios como é o caso da ansiedade somática. $\mathrm{Na}$ realidade as relações obtidas não se enquadram em qualquer referencial teórico, daí não serem interpretáveis.

Os resultados não suportaram empiricamente o modelo estrutural proposto por Ntoumanis e Biddle (1998), no qual a orientação para o ego influenciava a ansiedade (cognitiva e somática) através da autoconfiança.

Face aos resultados obtidos sugerimos que estudos futuros investiguem a validade e pertinência do modelo avaliado mas em comparação com outros alternativos e recorrendo a amostras de grandes dimensões e homogéneas e com o cuidado de acautelar a integração das múltiplas variáveis que a literatura já demonstrou estarem na base do bem-estar dos indivíduos. 


\section{Agradecimentos:}

$\mathrm{O}$ presente estudo somente foi possível devido às colaborações dos atletas que concordaram em participar como sujeitos da investigação.

\section{Conflito de Interesses:}

Nada declarado.

\section{Financiamento:}

Nada declarado.

\section{REFERÊNCIAS}

Albuquerque, A. S. \& Tróccoli, B. T. (2004). desenvolvimento de uma escala de bem-estar subjetivo. Psicologia: Teoria e Pesquisa, 20(2), 153-164.

Ames, C. (1992). Classrooms: goals, structure, and student motivation. Journal of Educational Psychology, 84, 261-271.

Bentler, P., \& Bonnet, D. (1980). Significance tests and goodness of fit in the analysis of covariance structures. Psychological Bulletin, 88, 588-606. doi: 10.1037/0033-2909.88.3.588

Browne, M. W., \& Cudek, R. (1993). Alternate ways of assessing model fit. In K. A. Bollen \& J. S. Long (Eds.), Testing structural equation models (pp. 136-162). Newbury Park, CA: Sage.

Cerin, E. (2004). Predictors of competitive anxiety direction in male Tae Kwon Do practitioners: A multilevel mixed idiographic/nomothetic interactional approach. Psychology of Sport and Exercise, 5, 497-516. doi:10.1016/S14690292(03)00041-4

Coelho, E., Vasconcelos-Raposo, J., \& Mahl, A. (2010). Confirmatory factorial analysis of the Brazilian version of the Competitive State Anxiety Inventory-2 (CSAI-2). Spanish Journal of Psychology, 13(1) 453-460.

Cox, R. H., Martens, M., \& Russell, W. D. (2003). Measuring anxiety in athletics: The revised Competitive State Anxiety Inventory-2. Journal of Sport \& Exercise Psychology, 25, 519-533.

Craft, L. L., Magyar, T. M., Becker, B. J., \& Feltz, D. L. (2003). The relationship between the Competitive State Anxiety Inventory-2 and sport perfor- mance: A meta-analysis. Journal of Sport and Exercise Psychology, 25, 44-65.

Diener, E., Emmons, R. A., Larsen, R. J., \& Griffin, S. (1985). The satisfaction with life scale. Journal of Personality Assessment, 49, 71-75.

Duda, J. L. (1989). Relationship between task and ego orientation and the perceived purposes of sport among high school athletes. Journal of Sport \& Exercise Psychology, 11, 318-335.

Duda, J. \& Nicholls, J. (1992). Dimensions of achievement motivation in schoolwork and sport. Journal of Educational Psychology, 84, 290-299.

Duda, J., Chi, L., Newton, M., Walling, M., \& Catley, D. (1995). Task and ego orientation and intrinsic motivation in sport. International Journal of Sport Psychology, 26(1), 40-63.

Duncan, T. E., \& Stoolmiller, M. (1993). Modeling social and psychological determinants of exercise behaviors via structural equation systems. Research Quarterly for Exercise and Sport, 64, 1-16.

Dweck, C. (1986). Motivational processes affecting learning. American Psychologist, 41, 1040-1048.

Dweck, C. S., \& Leggett, E. L. (1988). A social-cognitive approach to motivation and personality. Psychological Review, 95, 256-273.

Elliot, E. S, \& Dweck, C. S. (1988). Goals: An approach to motivation and achievement. Journal of Personality and Social Psychology, 54, 5-12.

Fernandes, H. \& Vasconcelos-Raposo, J. (2010). Análise fatorial confirmatória do TEOSQp. Psicologia Reflexão e Crítica, 23, 92-101. doi: 10.1590/S0102-79722010000100012

Fernandes, M. G., Vasconcelos-Raposo, J., \& Fernandes, H. (no prelo). Propriedades psicométricas do CSAI-2 em atletas brasileiros. Psicologia: Reflexão e Crítica.

Galand, B., Boudrenghien, G., \& Rose, A. (in press). Buts personnels, orientations motivationnelles et bien-être subjectif: Effets indépendants ou médiatisés ? Canadian Journal of Behavioural Science. doi: 10.1037/a0024389

Gouveia, V. V., Milfont, T. L., Fonseca, P. N., \& Coelho, J. A. (2009). Life satisfaction in Brazil: Testing the psychometric properties of the 
Satisfaction With Life Scale (SWLS) in Five Brazilian Samples. Social Indicators Research, 90, 267-277. doi: 10.1007/s11205-008-9257-0

Hair, J., Anderson, R, Tatham, R., \& Black, W. (2005). Análise multivariada de dados. Porto Alegre: Bookman.

Hall, H. K., Kerr, A. W., \& Matthews, J. (1998). Precompetitive anxiety in sport: The contribution of achievement goals and perfectionism. Journal of Sport $\mathcal{E}$ Exercise Psychology, 20(2), 194-217.

Heazlewood, I., \& Burke, S. (2011). Self-efficacy and its relationship to selected sport psychological constructs in the prediction of performance in ironman triathlon. Journal of Human Sport \& Exercise, 6(60), 328-350. doi: 10.4100/ jhse.2011.62.14

Hefferson, K., \& Boniwell, I. (2011). Positive psychology: Theory, research and applications. Berkshire: MacGraw-Hill.

Hu, L.-T., \& Bentler, P. M. (1999). Cut-off criteria for fit indices in covariance structure analysis: Conventional criteria versus new alternatives. Structural Equation Modeling, 6, 1-55. doi: 10.1080/10705519909540118

Jones, G., Hanton, S., and Swain, A. B. J. (1994). Intensity and interpretation of anxiety symptoms in elite and non-elite sports performers. Personality and Individual Differences, 17, 657-663. doi: 10.1016/0191-8869(94)90138-4

Jones, G., Swain, A. B. J., \& Harwood, C. (1996). Positive and negative affect as predictors of competitive anxiety. Personality and Individual Differences, 20, 109-114.

Jones, J. G. (1995). More than just a game: Research developments and issues in competitive anxiety in sport. British Journal of Psychology, 85, 449-478.

Jöreskog, K. G., \& Sörbom, D. (1989). LISREL 7: User's reference guide. Mooresville, IN: Scientific Software.

Martens, R., Vealey, R. S., \& Burton, D. (1990). Competitive anxiety in sport. Champaign, IL: Human Kinetics.

Mellalieu, S.D. \& Hanton, S. (2008). Facilitating anxiety: Myth or mislabeled? The relationship between interpretations of competitive anxiety symptoms and positive affective states In. M.P Simmons \& L.A. Foster (Eds.), Sport and exercise psychology research advances (pp 53-83). Hauppauge, NY: Nova Science.

Nicholls, J. G. (1989). The competitive ethos and demogratic education. Cambridge, MA: Harvard University Press.

Ntoumanis N, \& Biddle S. J. H. (1998). The relationship between competitive anxiety, achievement goals, and motivational climates. Research Quarterly for Exercise and Sport, 69(2), 176-187.

Ntoumanis N, \& Biddle S. J. H. (1999). Affect and achievement goals in physical activity: A meta-analysis. Scandinavian Journal of Medicine Sciences Sports, 9, 315-332.

Nunez, J., León, J., González, V. \& Martín-Albo, J. (2011). Propuesta de un modelo explicativo del bienestar psicológico en el contexto Deportivo. Revista de Psicología del Deporte, 20(1), 223-242.

Robazza, C., Bortoli, L., Nocini, F., Moser, G., \& Arslan, C. (2000). Normative and idiosyncratic measures of positive and negative affect in sport. Psychology of Sport and Exercise, 1, 103-116.

Roberts, G. C. (1992). Motivation in sport and exercise: Conceptual constraints and convergence. In G. C. Roberts (Ed.), Motivation in sport and exercise (pp. 3-29). Champaign, IL: Human Kinetics.

Roberts, G. C. (2001). Understanding the dynamics of motivation in physical activity: The influence of achievement goals on motivational processes. In G. C. Roberts (Ed.), Advances in motivation in sport and exercise (pp. 1-50). Champaign, Il: Human Kinetics.

Rodrigues, A. D., Lázaro, J. P., Fernandes, H. M., \& Vasconcelos-Raposo, J. (2009). Caracterização dos níveis de negativismo, activação, autoconfiança e orientações motivacionais de alpinistas. Motricidade, 5(2), 63-86.

Salmela-Aro, K., Nurmi, J., Saisto, T., \& Halmesmki, E. (2001). Goal construction and depressive symptoms during transition to motherhood: Evidence from two longitudinal studies. Journal of Personality and Social Psychology, 81, 1144-1159. 
$18 \mid$ M.G. Fernandes, J. Vasconcelos-Raposo, H.M. Fernandes

Seligman, M. E., \& Csikszentmihalyi, M. (2000). Positive psychology: An introduction. American Psychologist, 55(1), 5-14.

Tello, F. P. H., Martínez, L. M., Núñez, M. L., \& Calvo, T. G. (2010). A structural model of goal orientation in sports: Personal and contextual variables. The Spanish Journal of Psychology, 13(1), 257-266.

Ullman, J. B. (2001). Structural equation modeling. In B. G. Tabachnick \& L. S. Fidell (Eds.), Using multivariate statistics (4a ed., pp. 653-771). Needham Heights, MA: Allyn \& Bacon.

Vasconcelos-Raposo, J., \& Mahl, A. (2005). Orientação cognitiva de atletas profissionais de futebol do Brasil. Motricidade, 1 (4), 253-265.
Watson, D. (1988). Intraindividual and interindividual analyses of positive and negative affect: Their relations to health complaints, perceived stress, and daily activities. Journal of Personality and Social Psychology, 54, 1020-1030.

Watson, D., \& Clark, L. A. (1984). Negative affectivity: The disposition to experience aversive emotional states. Psychological Bulletin, 96, 219-235.

Woodman, T., \& Hardy, L. (2001). Stress and anxiety. In R. Singer, H. A. Hausenblas, \& C. M. Janelle (Eds.), Handbook of research on sport psychology (pp. 290-318). New York: Wiley.

(co) BY-NC Todo o conteúdo da revista Motricidade está licenciado sob a Creative Commons, exceto quando especificado em contrário e nos conteúdos retirados de outras fontes bibliográficas. 